\title{
Adjpocytokine responses to acute exercise in athletes with olfifierent body fat content and sedentary controls
}

Authors: Marina Djelic a, Sanja Mazic ${ }^{a}$,Biljana Lazovic ${ }^{b}$, Dejan Zikic ${ }^{c}$, Mirjana Sumarac-Dumanovic ${ }^{d}$, Dragan Micic ${ }^{d}$

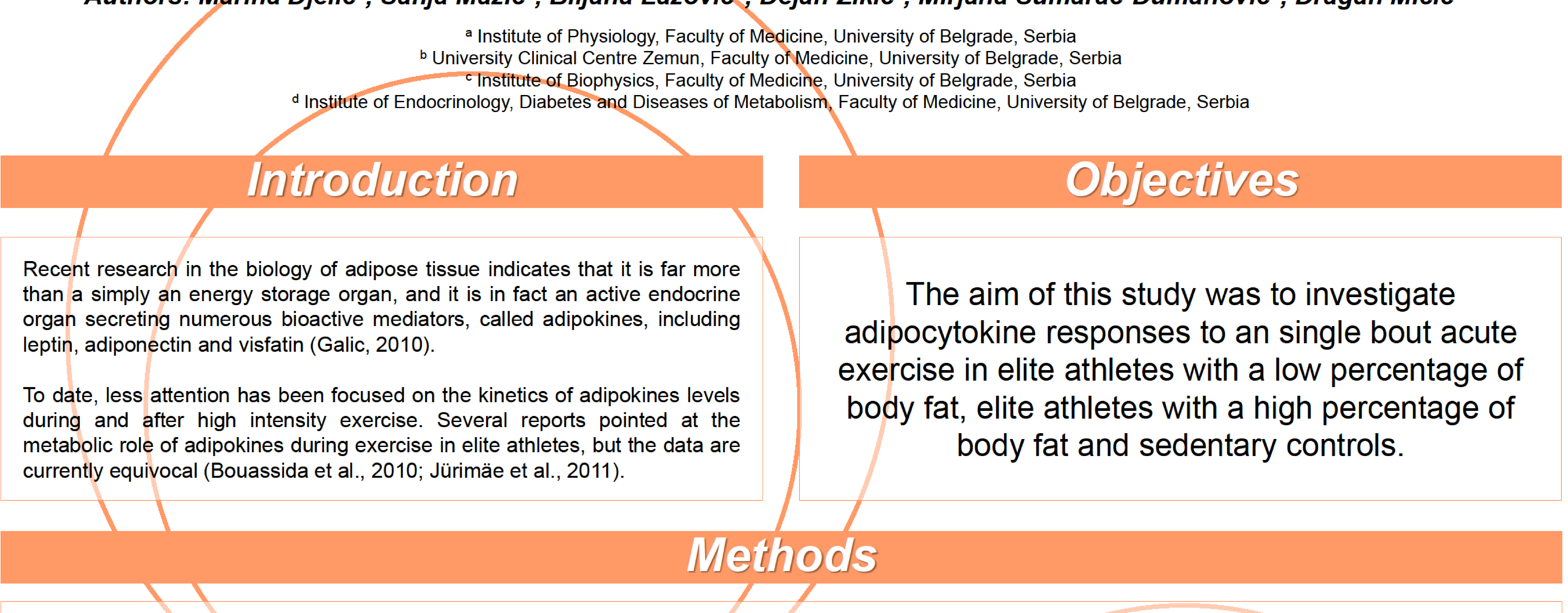

Sixteen athletes with low percentage of body fat (volleyball players, low fat athletes group, LFAG), fifteen athletes with high percentage of body fat (water polo players, high fat athletes group, HFAG) and fifteen sedentary subjects participated in this study (age (years) $20 \pm 2 ; 20 \pm 2 ; 20 \pm 1$, respectively).

All subjects were exposed to:

1. Anthropometric measurements:

- Body mass $(\mathrm{kg})$ - digital scale on a flat floor, participants wearing minimal clothing

- Body height $(\mathrm{cm})$ - tape measure and a set square while the subjects were standing straight against a wall without shoes

- $\quad$ BMI was calculated using body weight (in kilograms) divided by body height squared (in meters)

- Body fat percentage was calculated using a digital body fat scale Tanita

2. exercise test on treadmill in order to examine acute changes of adipocytokines.

Blood samples were obtained at baseline levels, immediately after the exercise test and 30 minutes after recovery. Separated serum or plasma were used for hormone (leptin, adiponectin and visfatin) ELISA analysis.

\section{Resultis}

Table 1. Anthropometric characteristic of groups

\begin{tabular}{|c|c|c|c|c|}
\hline & \multicolumn{2}{|c|}{ Athletes } & \multirow[b]{2}{*}{$\begin{array}{l}\text { Control } \\
(n=16)\end{array}$} & \multirow[t]{2}{*}{$\mathbf{p}$} \\
\hline & $\begin{array}{l}\text { LFAG } \\
(n=16)\end{array}$ & $\begin{array}{l}\text { HFAG } \\
(n=15)\end{array}$ & & \\
\hline BM (kg) & $84.3 \pm 6.9$ & $99.0 \pm 11.6$ & $90.2 \pm 10.4$ & $+\&$ \\
\hline $\mathrm{BH}(\mathrm{cm})$ & $196.3 \pm 5.5$ & $194.8 \pm 4.4$ & $184.3 \pm 6.8$ & $*_{+}$ \\
\hline BMI $\left(\mathrm{kg} / \mathrm{m}^{2}\right)$ & $21.9 \pm 1.9$ & $26.1 \pm 2.9$ & $26.5 \pm 1.9$ & *\& \\
\hline BF\% (\%) & $8.4 \pm 2.1$ & $16.8 \pm 2.6$ & $17.5 \pm 2.9$ & *\& \\
\hline
\end{tabular}

Data are means \pm SD; LFAG, low fat athletes group; HFAG, high fat athletes group; BM, body mass; $\mathrm{BH}$, body high; $\mathrm{BMI}$, body mass index; $\mathrm{BF} \%$, body fat percentage; ${ }^{*}, \mathrm{p}<0.05$ between LFAG and controls; +, $p<0.05$ between HFAG and controls; \&, $p<0.05$ between athletes

\section{Resultis}

Figure 1. Leptin response to acute exercise Figure 2. Visfatin response to acute exercise

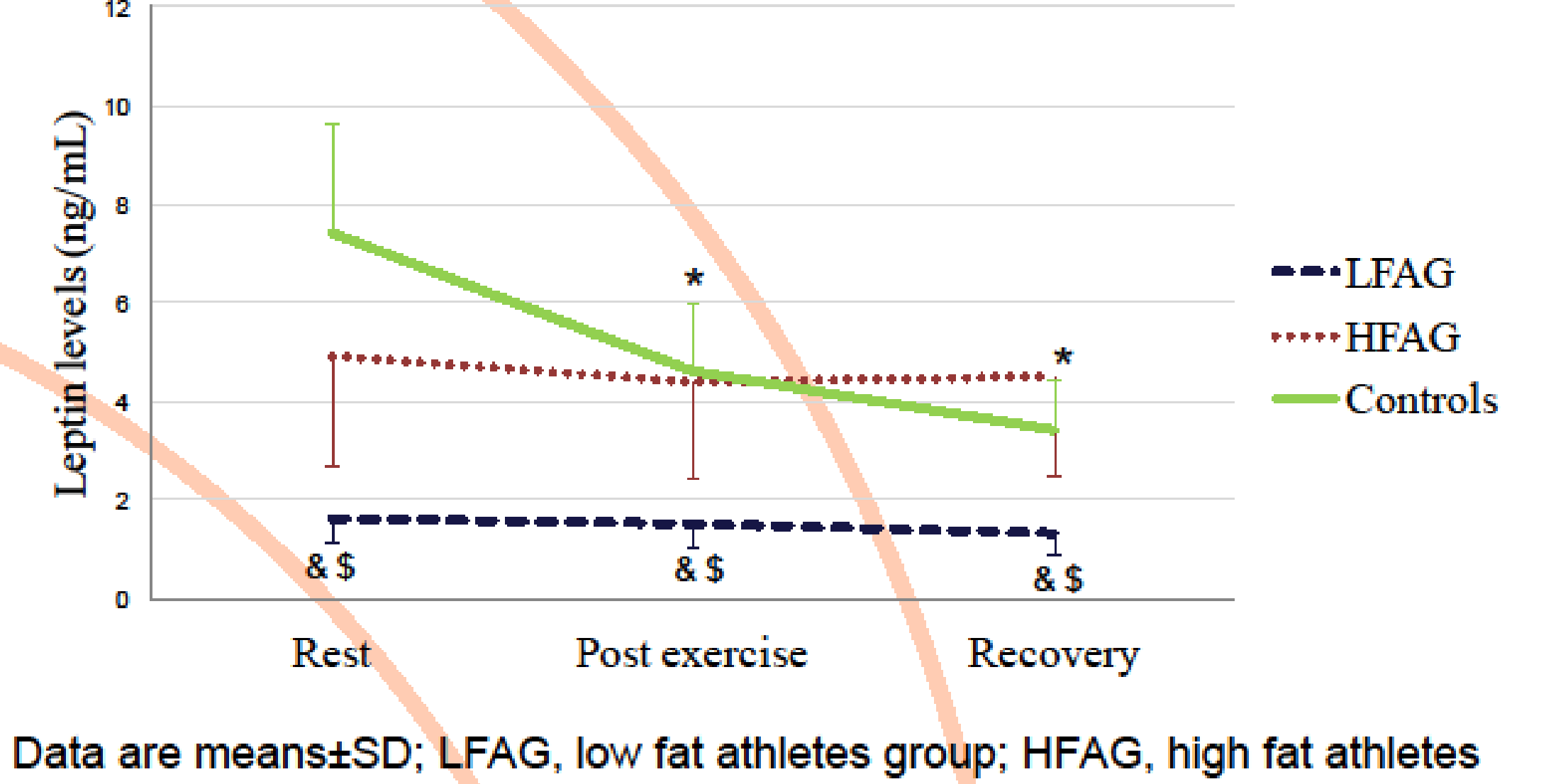

Data are meanstSD; LFAG, low fat athletes group; HFAG, high fat athletes
group; * , $p<0.05$, in compare with rest; $\&, p<0.05$ in compare to controls; $\$$.

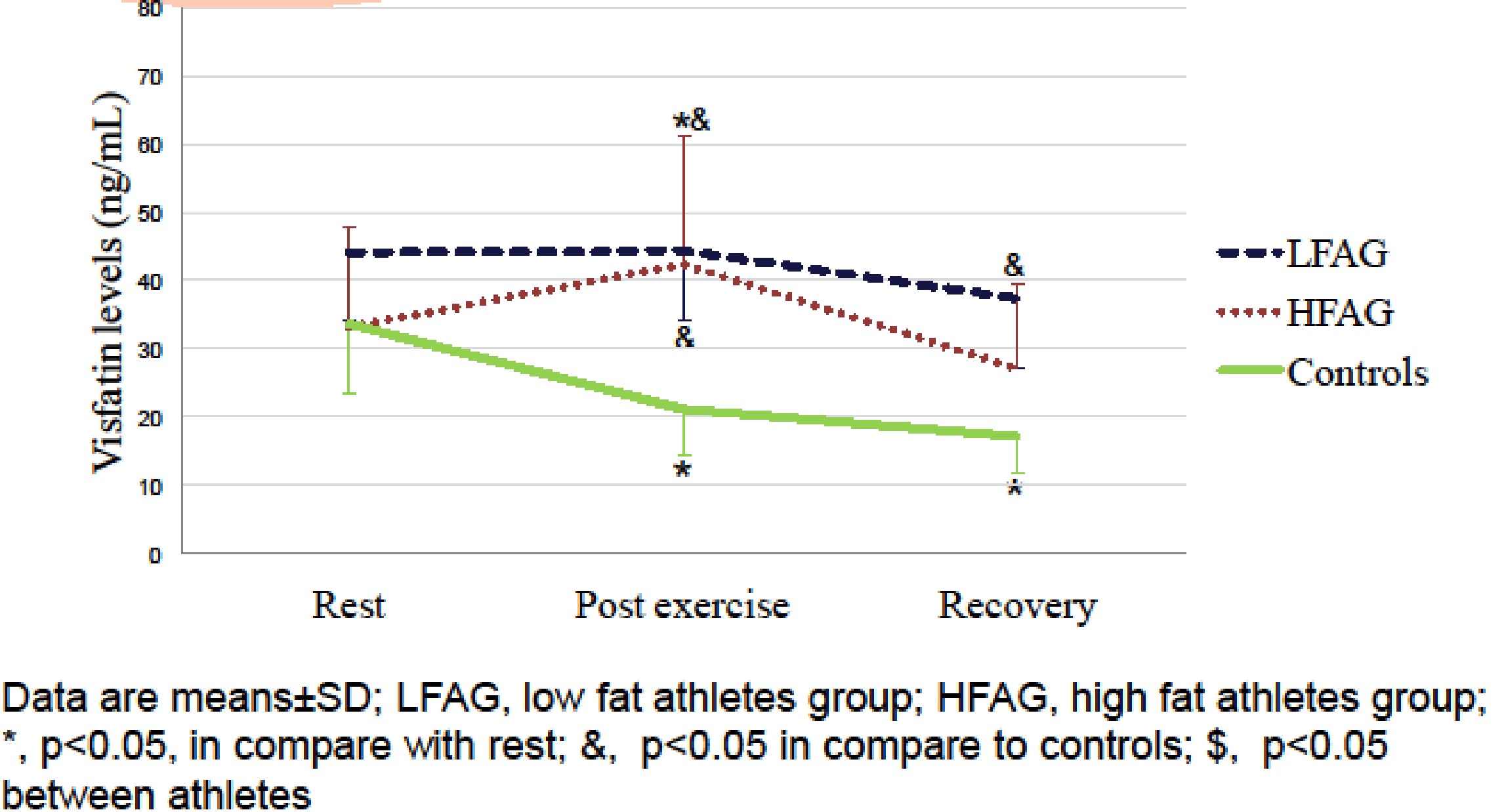

Data are means $\pm S D ;$ LFAG, low fat athletes group; HFAG, high fat athletes group;
$*, p<0.05$, in compare with rest; $\&, p<0.05$ in compare to controls; $\$, p<0.05$

In athletes in LFAG, baseline leptin concentration was significantly lower, but adiponectin and visfatin concentrations were significantly higher, compared to sedentary controls and athletes in HFAG $(p<0.05$, all).

There were no significant post exercise or recovery changes in adiponectin concentration $(p>0.05)$.

\section{Conclusions}

Our findings show leptin and visfatin levels, but not adiponectin respond to acute exercise. Acute exercise elicited an inverse visfatin response in athletes in HFAG and controls. Also, these results suggest that leptin is altered after acute exercise only in sedentary individuals. 